OPEN ACCESS

Edited by:

John D. Imig,

Medical College of Wisconsin,

United States

Reviewed by:

Ulrike Garscha,

Friedrich Schiller University Jena,

Germany

Xiang Fang,

The University of Texas Medical Branch at Galveston, United States

${ }^{*}$ Correspondence:

Qian Ren

renfflnm@gmail.com;

renfflnm@126.com

Specialty section:

This article was submitted to

Translational Pharmacology,

a section of the journal

Frontiers in Pharmacology

Received: 10 November 2018

Accepted: 03 April 2019

Published: 24 April 2019

Citation:

Ren Q (2019) Soluble Epoxide Hydrolase Inhibitor: A Novel Potential

Therapeutic or Prophylactic Drug for

Psychiatric Disorders.

Front. Pharmacol. 10:420

doi: 10.3389/fphar.2019.00420

\section{Soluble Epoxide Hydrolase Inhibitor: A Novel Potential Therapeutic or Prophylactic Drug for Psychiatric Disorders}

\author{
Qian $\operatorname{Ren}^{1,2 *}$ \\ ${ }^{1}$ Department of Human Anatomy, Hebei Medical University, Shijiazhuang, China, ${ }^{2}$ Center of Stem Cell and Immune Cell \\ Research, Institute of Medical and Health Science, Hebei Medical University, Shijiazhuang, China
}

Psychiatric disorders, including depression and schizophrenia, affect millions of individuals worldwide. However, the precise neurobiology of psychiatric disorders remains unclear. Accumulating evidence suggests that various inflammatory processes play a key role in depression and schizophrenia, and that anti-inflammatory drugs exert a therapeutic effect in patients with psychiatric disorders. Epoxyeicosatrienoic acids (EETs) and epoxydocosapentaenoic acids (EDPs) have potent anti-inflammatory properties. These mediators are broken down into their corresponding diols by soluble epoxide hydrolase (sEH), and inhibition of sEH enhances the anti-inflammatory effects of EETs. Therefore, sEH may play a key role in inflammation, which is involved in psychiatric disorders. Recent studies have shown that abnormal levels of sEH may be involved in the pathogenesis of certain psychiatric diseases, and that sEH inhibitors exhibit antidepressant and antipsychotic activity. The present review discusses the extensive evidence supporting $\mathrm{sEH}$ as a therapeutic target for psychiatric diseases, and the clinical value of sEH inhibitors as therapeutic or prophylactic drugs.

Keywords: depression, antidepressant, schizophrenia, soluble epoxide hydrolase, inflammation

\section{INTRODUCTION}

Depression and schizophrenia are severe and chronic debilitating psychiatric diseases that affect millions of individuals worldwide, with $>300$ million individuals of all ages affected by depression and nearly 800,000 dying each year from suicide (World Health Organization, 2018a). Similarly, schizophrenia affects $>21$ million individuals, and the probability of death in this patient population is 2 to 3 times higher than that in the general population (World Health Organization, 2018b). Although current clinical antidepressants and antipsychotics have been shown to be effective in the treatment of depression and schizophrenia, at least one-third of individuals with depression do not fully respond to medications, and antipsychotics have no beneficial effects on negative symptoms and cognitive impairments (Hashimoto, 2014; Steinert et al., 2014; Biesheuvel-Leliefeld et al., 2015; Guidi et al., 2016). These limitations highlight the need for a new class of antidepressants and antipsychotics, particularly for patients with treatment-resistant disease. 
There is ample evidence that inflammation plays a central role in the pathophysiology of depression and schizophrenia (Dantzer et al., 2008; Potvin et al., 2008; Girgis et al., 2014; Gold, 2015; Hashimoto, 2015; Steullet et al., 2016; Marques et al., 2018; Swardfager et al., 2018). In patients not undergoing antidepressant therapy, meta-analyses have reported that blood levels of proinflammatory cytokines, including tumor necrosis factor- $\alpha$ (TNF- $\alpha$ ) and interleukin 6 (IL-6), are significantly higher than in healthy controls (Dowlati et al., 2010; Young et al., 2014; Haapakoski et al., 2015; Strawbridge et al., 2015). Moreover, studies of postmortem brain samples revealed increased proinflammatory cytokine gene expression in the prefrontal cortex (PFC) of individuals with a history of depression (Dean et al., 2010; Shelton et al., 2011). Studies using animal models of depression have reported lipopolysaccharide (LPS)-induced depression-like behavior and dendritic changes (Zhang et al., 2015, 2016). In studies investigating schizophrenia, the levels of proinflammatory cytokines, such as TNF- $\alpha$, and IL-6, were significantly elevated in the serum and cerebrospinal fluid (Sasayama et al., 2013; Upthegrove et al., 2014; Schwieler et al., 2015; Dickerson et al., 2016). Postmortem studies and metaanalyses have demonstrated increased microglia density and activity in patients with schizophrenia (van Kesteren et al., 2017; Marques et al., 2018). Several studies have demonstrated that anti-inflammatory drugs exhibit antipsychotic activity in animal models of schizophrenia (Shirai et al., 2012, 2015). Collectively, these investigations demonstrate that inflammation is likely closely related to depression and schizophrenia, and that anti-inflammatory drugs could effectively improve the symptoms of depression and schizophrenia.

\section{SOLUBLE EPOXIDE HYDROLASE AND THE ARACHIDONIC PATHWAY}

Extensive evidence suggests that biological substances in the arachidonate cascade, such as enzymes and eicosanoid metabolites, are involved in the etiology and pathology of inflammatory disease. Polyunsaturated fatty acids (PUFAs), such as arachidonic acid (AA), docosahexaenoic acid (DHA), and eicosapentaenoic acid (EPA), are metabolized by cyclooxygenases (COXs), lipoxygenases (LOXs), and cytochrome P450s (CYPs) (Figure 1; Imig and Hammock, 2009; Imig, 2012, 2018; Morisseau and Hammock, 2013). The COX and LOX pathways lead to the production of prostaglandins, leukotrienes, and hydroxyeicosatetraenoic acids (HETEs). These lipid mediators are involved in pro-inflammatory processes, while lipoxins synthesized from AA by LOX play an important role in the resolution of inflammation (Serhan and Savill, 2005; Serhan, 2017a,b). In contrast, the CYP pathway is involved in both the production of pro-inflammatory lipid mediators and anti-inflammatory lipid mediators. The CYP hydroxylases lead to 20-HETE (pro-inflammatory mediator), and CYP epoxygenases lead to epoxyeicosatrienoic acids (EETs) (anti-inflammatory mediator). Through catalyzing the epoxidation of PUFAs, such as $\mathrm{AA}$, the epoxygenase CYP enzymes generate four regioisomeric EETs, including 5,6-EET, 8,9-EET, 11,12-EET, and 14,15-EET. However, the EETs are metabolized by soluble epoxide hydrolase $(\mathrm{sEH})$ and converted into their corresponding diols (dihydroxyeicosatrienoic acids [DHETs]), and these molecules are considered to be less biologically active than their parent versions. Therefore, it is probable that sEH plays a role in the pathogenesis of several diseases caused by inflammation (Imig, 2005, 2012, 2016, 2018; Iliff et al., 2010; Morisseau and Hammock, 2013; Wagner et al., 2014, 2017; Zhang et al., 2014; Hashimoto, 2016).

\section{ROLE OF SEH IN PSYCHIATRIC DISORDERS}

As discussed above, inflammation is associated with psychiatric disorders such as depression and schizophrenia, and $\mathrm{sEH}$ plays a role in the pathogenesis of inflammatory-related diseases. Therefore, it is possible that sEH contributes to the pathophysiology of these disorders. Recently, our study

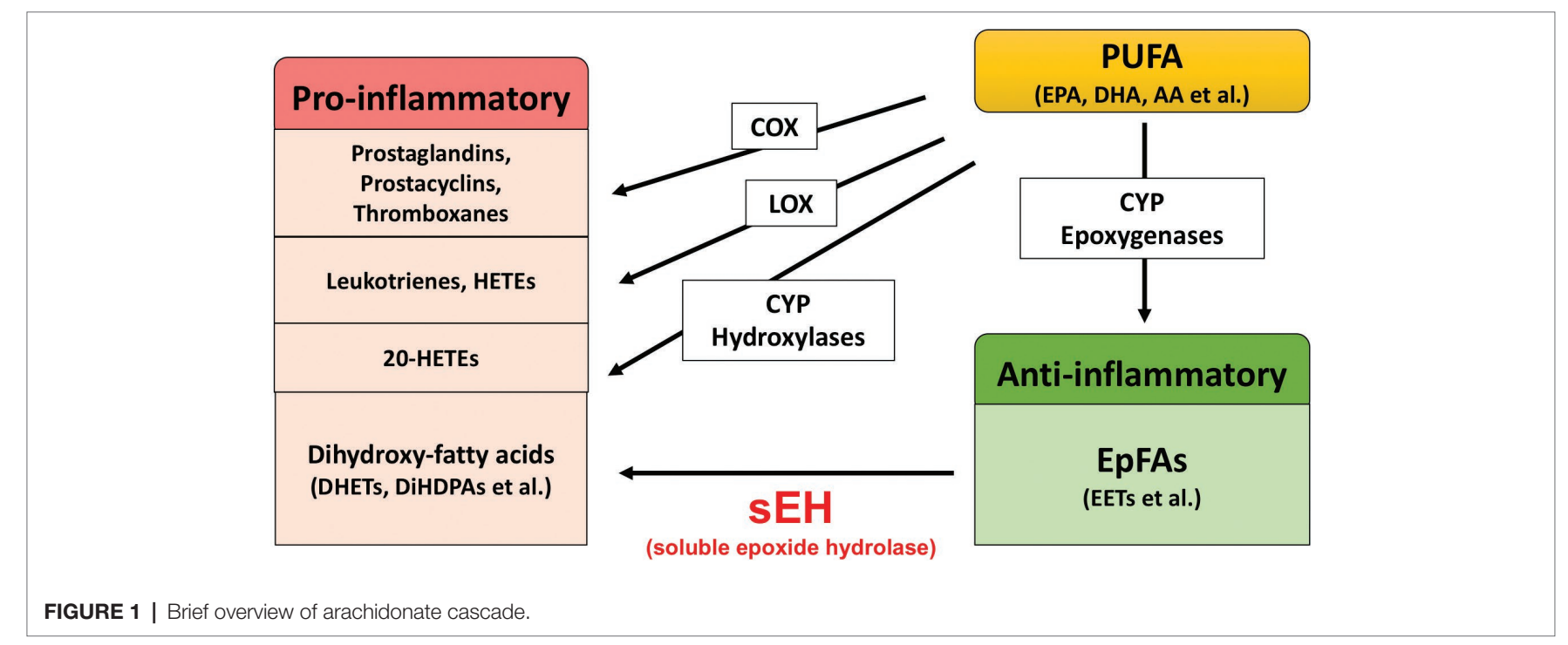


of postmortem brains revealed that the protein levels of sEH in the parietal cortex of patients with major depressive disorder, schizophrenia, and bipolar disorder were significantly elevated compared with those in the parietal cortex of healthy individuals (Ren et al., 2016). In studies involving mouse models, the brain, after inflammation or chronic social defeat stress, exhibited increased expression of $\mathrm{sEH}$ in the PFC and hippocampus. Meanwhile, after inflammation or social defeat stress, mice exhibited increased immobility time in tail suspension and forced swim tests, and decreased sucrose preference. These phenotypes were defined as depression-like behavior in mice (Duman and Monteggia, 2006; Dantzer et al., 2008). Studies involving behavioral tests in animal models have suggested that the sEH inhibitor 1-[1-propionylpiperidin4-yl]-3-[4-(trifluoromethoxy) phenyl] urea (TPPU) had antidepressant effects because the inflammation and chronic social defeat stress-induced depression-like behavior was prevented by oral administration or chronic intake of TPPU (Ren et al., 2016). As such, TPPU could produce antidepressant effects in inflammation model of depression because standard antidepressants, such as selective serotonin reuptake inhibitors and serotonin norepinephrine reuptake inhibitors, do not demonstrate therapeutic effects in such models (Zhang et al., 2015). Moreover, the use of TPPU lowered serum TNF- $\alpha$ levels in LPS-treated mice but not control mice. Additionally, in these experiments, both TPPU and 14,15-EET potentiated nerve growth factor (NGF)-induced neuronal outgrowth in PC12 cells. Consistent with the pharmacological inhibition of $\mathrm{sEH}$, despite experiencing chronic social defeat stress, sEH knockout mice did not exhibit depression-like behavior. It is likely that the deletion of the $\mathrm{sEH}$ gene conferred resilience to social defeat stress. Additionally, investigation of brain-derived neurotrophic factor-tropomyosin receptor kinase B (BDNF-TrkB) signaling protein expression in $\mathrm{SEH}$ knockout mice brains revealed that BDNF and p-TrkB-toTrkB protein ratios were elevated in the PFC and hippocampus. Consistent with BDNF-TrkB signaling, the protein levels of glutamate receptor subunit (GluA1) and postsynaptic density protein (PSD-95), which are synaptogenesis biomarkers, were elevated in the PFC and hippocampus of sEH knockout mice. This could infer that deletion of sEH resulted in resilience to chronic social defeat stress via increased BDNFTrkB signaling and synaptogenesis. Furthermore, data reported by other groups are in agreement with these results. Wu et al. reported that the $\mathrm{sEH}$ inhibitor TPPU decreased depression-like behavior in the novelty-suppressed feeding test, which is a test of stress-induced anxiety/depression (Wu et al., 2017). Moreover, treatment with TPPU elevated the expression of BDNF in the mouse hippocampus and PC12 cells, and the antidepressant effect of TPPU was blocked by a BDNF-TrkB signal pathway antagonist (Wu et al., 2017, 2019). This evidence suggests that BDNF is necessary for the antidepressant effects of TPPU. Collectively, these findings highlight a key function of sEH in the etiology and pathology of depression, and for its inhibitors as potential therapeutic or prophylactic drugs for depression (Ren et al., 2016; Hashimoto, 2016).
As mentioned above, patients with schizophrenia exhibit higher sEH protein levels in the parietal cortex than controls. Meanwhile, another study investigating alterations of eicosanoids in the serum of patients with schizophrenia reported that 11,12-DHETs and 14,15-DHETs were increased in patients compared with controls and were decreased post-treatment (Wang et al., 2018). This evidence suggested that EET and its metabolic enzyme sEH may play a role in schizophrenia, and studies using animal models have provided strong supportive data. Ma et al. (2013) investigated the effects of AS2586114, a potent sEH inhibitor, in an animal model of schizophrenia. In a phencyclidine (PCP)induced model of schizophrenia, a single oral administration of AS2586114 attenuated PCP-induced hyperlocomotion in a dose-dependent manner. Furthermore, AS2586114 also improved PCP-induced prepulse inhibition deficits in a dose-dependent manner. In addition, AS2586114 exhibited a similar effect to the atypical antipsychotic drug clozapine in PCP-induced behavioral abnormalities (Ma et al., 2013). These studies suggest the therapeutic potential of sEH inhibitors for schizophrenia. However, the precise mechanism by which $\mathrm{sEH}$ inhibitors diminish PCP-induced acute behavioral effects in mice remains unclear. Nevertheless, some studies have provided valuable clues. Ribeiro et al. reported that omega-3 PUFAs (n3 PUFAs), but not clozapine, prevented polyinosinic:polycytidylic acid (poly I:C)-induced deficits in BDNF (Ribeiro et al., 2019). Because decreased BDNF-TrkB signaling has been suggested to play a role in the pathophysiology of schizophrenia (Giovanoli et al., 2015; Han et al., 2016), and sEH inhibitors increase the level of BDNF, it appears that sEH may play a role in schizophrenia via regulation of the BDNFTrkB signaling pathway. There is ample evidence suggesting that oxidative stress also plays an important role in the pathophysiology of schizophrenia, and antioxidant agents have demonstrated antipsychotic effects in animal models of schizophrenia (Matsuzawa and Hashimoto, 2011; Reddy and Reddy, 2011; Yao and Keshavan, 2011; Shirai et al., 2012, 2015). Moreover, abnormalities in striatal dopamine levels are a hallmark of schizophrenia pathophysiology (Brisch et al., 2014; Nakao et al., 2019). Recently, Ren et al. (2018) reported that inhibition of sEH protected against MPTP (1-methyl-4-phenyl-1,2,3,6-tetrahydropyridine)-induced endoplasmic reticulum (ER) stress and oxidative stress in the brain. The immunoreactivity of $\mathrm{sEH}$ is present almost exclusively in astrocytes throughout the brain (Marowsky et al., 2009); however, deletion of the sEH gene suppressed MPTP-induced activation of microglia in the mouse striatum (Ren et al., 2018). Additionally, inhibition of sEH attenuated MPTP-induced dopaminergic dysfunction in the striatum (Ren et al., 2018). Other studies have consistently found that deletion of the sEH gene and pharmacological inhibition of sEH blocked MPTPinduced heme-oxygenase (HO-1) elevation (a redox-regulated protein) and caspase 12 activation (a hallmark of ER stress) (Huang et al., 2018). These findings indicate that sEH inhibitors are effective in attenuating MPTP-induced dopaminergic neurotoxicity, oxidative stress, and ER stress. Given the evidence described, there is a possibility that $\mathrm{sEH}$ inhibitors exert their antipsychotic properties by increasing EETs, modulating the BDNF-TrkB signaling pathway, protecting against oxidative stress, and improving dopaminergic dysfunction in the brain. 


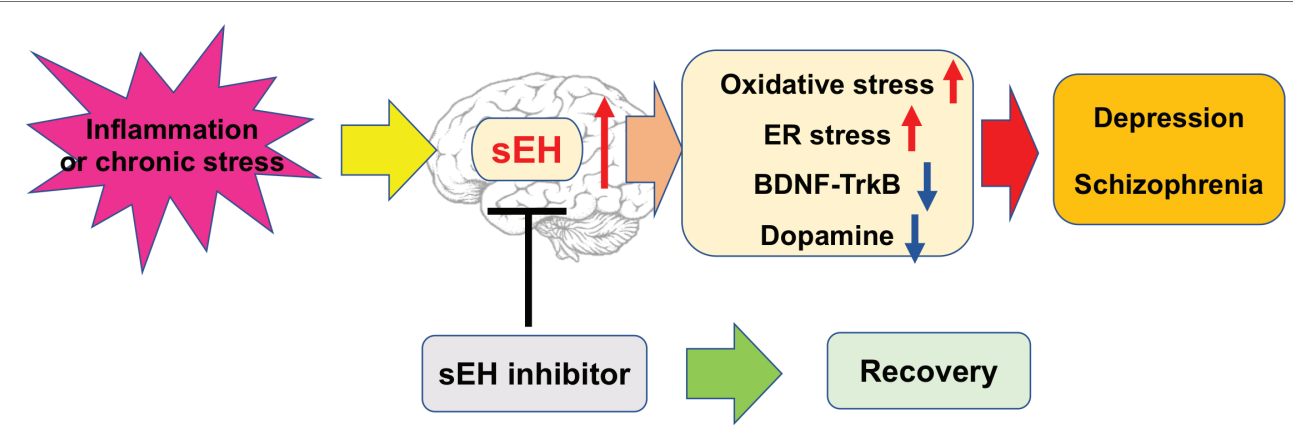

FIGURE 2 | Possible mechanisms of the role of sEH in depression and schizophrenia.

\section{DISCUSSION}

In this minireview, we highlighted recent studies that have demonstrated the potential of $\mathrm{sEH}$ as a therapeutic target in psychiatric disorders. Crucial data from Ren et al. (2018) and $\mathrm{Ma}$ et al. (2013) demonstrate that protein levels of $\mathrm{sEH}$ in the brains of depressed and schizophrenic patients are higher than in controls. Additionally, sEH protein levels are elevated in the brains of mice with a depression-like phenotype. These data suggest that increased levels of sEH in the brain cause enhanced metabolism of anti-inflammatory PUFA epoxides, such as EETs, EDPs, and epoxyeicosatetraenoic acids (EEQs), eventually leading to depressive symptoms. Furthermore, a single administration of $s E H$ inhibitor has been reported to prevent depression-like phenotypes in the inflammation and chronic social defeat stress models of depression. These important findings indicate that sEH inhibitors may have a rapid onset of antidepressive action, which is similar to the rapid-acting antidepressant ketamine, but without any observable side effects (Hashimoto, 2016). Moreover, a single administration of sEH inhibitor has also been reported to rescue PCP-induced behavioral abnormality in mice. These antipsychotic effects of sEH inhibitor are similar to those of the atypical antipsychotic drug clozapine. This is noteworthy because sEH inhibitors also may have a rapid onset of antipsychotic action without any observable side effects.

Several hypotheses have postulated that inflammation may play a causative role in depression and schizophrenia (Schiepers et al., 2005; Brown and Derkits, 2010; Brown and Meyer, 2018; Chen et al., 2019). However, how do inflammatory cytokines influence behavior? In the brain, inflammatory cytokines, such as TNF- $\alpha$, IL-6, and IL- $1 \beta$, are elevated in the brain during inflammation or chronic stress (Strawbridge et al., 2015; Menard et al., 2017). These cytokines have been implicated in the

\section{REFERENCES}

Biesheuvel-Leliefeld, K. E., Kok, G. D., Bockting, C. L., Cuijpers, P., Hollon, S. D., van Marwijk, H. W., et al. (2015). Effectiveness of psychological interventions in preventing recurrence of depressive disorder: meta-analysis and meta-regression. J. Affect. Disord. 174, 400-410. doi: 10.1016/j. jad.2014.12.016 activation of indoleamine-2,3-dioxygenase (IDO) and tryptophan dioxygenase (TDO), and promote the metabolization of tryptophan into formylkynurenine, a precursor of kynurenine. Furthermore, the metabolites of kynurenine, such as kynurenic acid and quinolinic acid, are involved in modulation of N-methyl-Daspartate receptor and $\alpha$-amino-3-hydroxy-5-methyl4-isoxazolepropionic acid receptor, and can also activate monoamine oxidase. Subsequently, these metabolites are implicated in dopamine synthesis, behavioral abnormalities, and regulation of neurotrophic and metabolic signaling through BDNF and mTOR (Schiepers et al., 2005; Calcia et al., 2016; Ghasemi et al., 2017; Lima Giacobbo et al., 2018; Price et al., 2018; Chen et al., 2019).

Collectively, these studies described a potential mechanism for sEH in the pathophysiology of depression and schizophrenia. This evidence suggests that sEH inhibitors exert an antidepressant and antipsychotic effect, and may attenuate the appearance of oxidative and ER stress, dysregulation of neurotrophic and dysfunction of dopaminergic neurons in the brain (Figure 2). Nevertheless, the precise mechanism of action of sEH inhibitors remains largely unknown, thus warranting more in-depth studies in the future.

\section{AUTHOR CONTRIBUTIONS}

QR wrote the manuscript and prepared the figures.

\section{FUNDING}

This study was supported by the Japan Society for the Promotion of Science (JSPS) KAKENHI grant (18k15439). 
Brown, A. S., and Meyer, U. (2018). Maternal immune activation and neuropsychiatric illness: a translational research perspective. Am. J. Psychiatry 175, 1073-1083. doi: 10.1176/appi.ajp.2018.17121311

Calcia, M. A., Bonsall, D. R., Bloomfield, P. S., Selvaraj, S., Barichello, T., and Howes, O. D. (2016). Stress and neuroinflammation: a systematic review of the effects of stress on microglia and the implications for mental illness. Psychopharmacology 233, 1637-1650. doi: 10.1007/s00213-016-4218-9

Chen, K. L., Cathomas, F., and Russo, S. J. (2019). Central and peripheral inflammation link metabolic syndrome and major depressive disorder. Physiology 34, 123-133. doi: 10.1152/physiol.00047.2018

Dantzer, R., O’Connor, J. C., Freund, G. G., Johnson, R. W., and Kelley, K. W. (2008). From inflammation to sickness and depression: when the immune system subjugates the brain. Nat. Rev. Neurosci. 9, 46-56. doi: 10.1038/nrn2297

Dean, B., Tawadros, N., Scarr, E., and Gibbons, A. S. (2010). Regionally-specific changes in levels of tumour necrosis factor in the dorsolateral prefrontal cortex obtained postmortem from subjects with major depressive disorder. J. Affect. Disord. 120, 245-248. doi: 10.1016/j.jad.2009.04.027

Dickerson, F., Stallings, C., Origoni, A., Schroeder, J., Katsafanas, E., Schweinfurth, L., et al. (2016). Inflammatory markers in recent onset psychosis and chronic schizophrenia. Schizophr. Bull. 42, 134-141. doi: 10.1093/schbul/sbv108

Dowlati, Y., Herrmann, N., Swardfager, W., Liu, H., Sham, L., Reim, E. K., et al. (2010). A meta-analysis of cytokines in major depression. Biol. Psychiatry 67, 446-457. doi: 10.1016/j.biopsych.2009.09.033

Duman, R. S., and Monteggia, L. M. (2006). A neurotrophic model for stressrelated mood disorders. Biol. Psychiatry 59, 1116-1127. doi: 10.1016/j. biopsych.2006.02.013

Ghasemi, M., Phillips, C., Fahimi, A., McNerney, M. W., and Salehi, A. (2017). Mechanisms of action and clinical efficacy of NMDA receptor modulators in mood disorders. Neurosci. Biobehav. Rev. 80, 555-572. doi: 10.1016/j. neubiorev.2017.07.002

Giovanoli, S., Notter, T., Richetto, J., Labouesse, M. A., Vuillermot, S., Riva, M. A., et al. (2015). Late prenatal immune activation causes hippocampal deficits in the absence of persistent inflammation across aging. J. Neuroinflammation 12:221. doi: 10.1186/s12974-015-0437-y

Girgis, R. R., Kumar, S. S., and Brown, A. S. (2014). The cytokine model of schizophrenia: emerging therapeutic strategies. Biol. Psychiatry 75, 292-299. doi: 10.1016/j.biopsych.2013.12.002

Gold, P. W. (2015). The organization of the stress system and its dysregulation in depressive illness. Mol. Psychiatry 20, 32-47. doi: 10.1038/mp.2014.163

Guidi, J., Tomba, E., and Fava, G. A. (2016). The sequential integration of pharmacotherapy and psychotherapy in the treatment of major depressive disorder: a meta-analysis of the sequential model and a critical review of the literature. Am. J. Psychiatry 173, 128-137. doi: 10.1176/appi. ajp.2015.15040476

Haapakoski, R., Mathieu, J., Ebmeier, K. P., Alenius, H., and Kivimäki, M. (2015). Cumulative meta-analysis of interleukins 6 and $1 \beta$, tumour necrosis factor $\alpha$ and $C$-reactive protein in patients with major depressive disorder. Brain Behav. Immun. 49, 206-215. doi: 10.1016/j.bbi.2015.06.001

Han, M., Zhang, J. C., Yao, W., Yang, C., Ishima, T., Ren, Q., et al. (2016). Intake of 7,8-dihydroxyflavone during juvenile and adolescent stages prevents onset of psychosis in adult offspring after maternal immune activation. Sci. Rep. 6:36087. doi: 10.1038/srep36087

Hashimoto, K. (2014). Targeting of NMDA receptors in new treatments for schizophrenia. Expert Opin. Ther. Targets 18, 1049-1063. doi: $10.1517 / 14728222.2014 .934225$

Hashimoto, K. (2015). Inflammatory biomarkers as differential predictors of antidepressant response. Int. J. Mol. Sci. 16, 7796-7801. doi: 10.3390/ijms16047796

Hashimoto, K. (2016). Soluble epoxide hydrolase: a new therapeutic target for depression. Expert Opin. Ther. Targets 20, 1149-1151. doi: 10.1080/14728222.2016.1226284

Huang, H. J., Wang, Y. T., Lin, H. C., Lee, Y. H., and Lin, A. M. (2018). Soluble epoxide hydrolase inhibition attenuates MPTP-induced neurotoxicity in the nigrostriatal dopaminergic system: involvement of a-synuclein aggregation and ER stress. Mol. Neurobiol. 55, 138-144. doi: 10.1007/ s12035-017-0726-9

Iliff, J. J., Jia, J., Nelson, J., Goyagi, T., Klaus, J., and Alkayed, N. J. (2010). Epoxyeicosanoid signaling in CNS function and disease. Prostaglandins Other Lipid Mediat. 91, 68-84. doi: 10.1016/j.prostaglandins.2009.06.004
Imig, J. D. (2005). Epoxide hydrolase and epoxygenase metabolites as therapeutic targets for renal diseases. Am. J. Physiol. Renal Physiol. 289, F496-F503. doi: 10.1152/ajprenal.00350.2004

Imig, J. D. (2012). Epoxides and soluble epoxide hydrolase in cardiovascular physiology. Physiol. Rev. 92, 101-130. doi: 10.1152/physrev.00021.2011

Imig, J. D. (2016). Epoxyeicosatrienoic acids and 20-hydroxyeicosatetraenoic acid on endothelial and vascular function. Adv. Pharmacol. 77, 105-141. doi: $10.1016 /$ bs.apha.2016.04.003

Imig, J. D. (2018). Prospective for cytochrome P450 epoxygenase cardiovascular and renal therapeutics. Pharmacol. Ther. 192, 1-19. doi: 10.1016/j. pharmthera.2018.06.015

Imig, J. D., and Hammock, B. D. (2009). Soluble epoxide hydrolase as a therapeutic target for cardiovascular diseases. Nat. Rev. Drug Discov. 8, 794-805. doi: $10.1038 / \mathrm{nrd} 2875$

Lima Giacobbo, B., Doorduin, J., Klein, H. C., Dierckx, R. A. J. O., Bromberg, E., and de Vries, E. F. J. (2018). Brain-derived neurotrophic factor in brain disorders: focus on neuroinflammation. Mol. Neurobiol. doi: 10.1007/s12035-018-1283-6

Ma, M., Ren, Q., Fujit, Y., Ishima, T., Zhang, J. C., and Hashimoto, K. (2013). Effects of AS2586114, a soluble epoxide hydrolase inhibitor, on hyperlocomotion and prepulse inhibition deficits in mice after administration of phencyclidine. Pharmacol. Biochem. Behav. 110, 98-103. doi: 10.1016/j. pbb.2013.06.005

Marowsky, A., Burgener, J., Falck, J. R., Fritschy, J. M., and Arand, M. (2009). Distribution of soluble and microsomal epoxide hydrolase in the mouse brain and its contribution to cerebral epoxyeicosatrienoic acid metabolism. Neuroscience 163, 646-661. doi: 10.1016/j.neuroscience.2009.06.033

Marques, T. R., Ashok, A. H., Pillinger, T., Veronese, M., Turkheimer, F. E., Dazzan, P., et al. (2018). Neuroinflammation in schizophrenia: meta-analysis of in vivo microglial imaging studies. Psychol. Med. 1-11. doi: 10.1017/ S0033291718003057

Matsuzawa, D., and Hashimoto, K. (2011). Magnetic resonance spectroscopy study of the antioxidant defense system in schizophrenia. Antioxid. Redox Signal. 15, 2057-2065. doi: 10.1089/ars.2010.3453

Menard, C., Pfau, M. L., Hodes, G. E., Kana, V., Wang, V. X., Bouchard, S., et al. (2017). Social stress induces neurovascular pathology promoting depression. Nat. Neurosci. 20, 1752-1760. doi: 10.1038/s41593-017-0010-3

Morisseau, C., and Hammock, B. D. (2013). Impact of soluble epoxide hydrolase and epoxyeicosanoids on human health. Annu. Rev. Pharmacol. Toxicol. 53, 37-58. doi: 10.1146/annurev-pharmtox-011112-140244

Nakao, K., Jeevakumar, V., Jiang, S. Z., Fujita, Y., Diaz, N. B., Pretell Annan, C. A., et al. (2019). Schizophrenia-like dopamine release abnormalities in a mouse model of NMDA receptor hypofunction. Schizophr. Bull. 45, 138-147. doi: 10.1093/schbul/sby003

Potvin, S., Stip, E., Sepehry, A. A., Gendron, A., Bah, R., and Kouassi, E. (2008). Inflammatory cytokine alterations in schizophrenia: a systematic quantitative review. Biol. Psychiatry 63, 801-808. doi: 10.1016/j. biopsych.2007.09.024

Price, J. B., Bronars, C., Erhardt, S., Cullen, K. R., Schwieler, L., Berk, M., et al. (2018). Bioenergetics and synaptic plasticity as potential targets for individualizing treatment for depression. Neurosci. Biobehav. Rev. 90, 212220. doi: 10.1016/j.neubiorev.2018.04.002

Reddy, R., and Reddy, R. (2011). Antioxidant therapeutics for schizophrenia. Antioxid. Redox Signal. 15, 2047-2055. doi: 10.1089/ars.2010.3571

Ren, Q., Ma, M., Ishima, T., Morisseau, C., Yang, J., Wagner, K. M., et al. (2016). Gene deficiency and pharmacological inhibition of soluble epoxide hydrolase confers resilience to repeated social defeat stress. Proc. Natl. Acad. Sci. USA 113, E1944-E1952. doi: 10.1073/pnas.1601532113

Ren, Q., Ma, M., Yang, J., Nonaka, R., Yamaguchi, A., Ishikawa, K. I., et al. (2018). Soluble epoxide hydrolase plays a key role in the pathogenesis of Parkinson's disease. Proc. Natl. Acad. Sci. USA 115, E5815-E5823. doi: $10.1073 /$ pnas. 1802179115

Ribeiro, B. M. M., Chaves Filho, A. J. M., Costa, D. V. D. S., de Menezes, A. T., da Fonseca, A. C. C., Gama, C. S., et al. (2019). N-3 polyunsaturated fatty acids and clozapine abrogates poly $\mathrm{I}$ :C-induced immune alterations in primary hippocampal neurons. Prog. Neuro-Psychopharmacol. Biol. Psychiatry 90, 186-196. doi: 10.1016/j.pnpbp.2018.11.022 
Sasayama, D., Hattori, K., Wakabayashi, C., Teraishi, T., Hori, H., Ota, M., et al. (2013). Increased cerebrospinal fluid interleukin-6 levels in patients with schizophrenia and those with major depressive disorder. J. Psychiatr. Res. 47, 401-406. doi: 10.1016/j.jpsychires.2012.12.001

Schiepers, O. J. G., Wichers, M. C., and Maes, M. (2005). Cytokines and major depression. Prog. Neuro-Psychopharmacol. Biol. Psychiatry 29, 201-217. doi: 10.1016/j.pnpbp.2004.11.003

Schwieler, L., Larsson, M. K., Skogh, E., Kegel, M. E., Orhan, F., Abdelmoaty, S., et al. (2015). Increased levels of IL-6 in the cerebrospinal fluid of patients with chronic schizophrenia-significance for activation of the kynurenine pathway. J. Psychiatry Neurosci. 40, 126-133. doi: 10.1503/jpn.140126

Serhan, C. N. (2017a). Discovery of specialized pro-resolving mediators marks the dawn of resolution physiology and pharmacology. Mol. Asp. Med. 58, 1-11. doi: 10.1016/j.mam.2017.03.001

Serhan, C. N. (2017b). Treating inflammation and infection in the 21st century: new hints from decoding resolution mediators and mechanisms. FASEB J. 31, 1273-1388. doi: 10.1096/fj.201601222R

Serhan, C. N., and Savill, J. (2005). Resolution of inflammation: the beginning programs the end. Nat. Immun. 6, 1191-1197. doi: 10.1038/ni1276

Shelton, R. C., Claibome, J., SidoryK-Wegrzynowicz, M., Reddy, R., Aschner, M., Lewis, D. A., et al. (2011). Altered expression of genes involved in inflammation and apoptosis in frontal cortex in major depression. Mol. Psychiatry 16, 751-762. doi: $10.1038 / \mathrm{mp} .2010 .52$

Shirai, Y., Fujita, Y., and Hashimoto, K. (2012). Effects of the antioxidant sulforaphane on hyperlocomotion and prepulse inhibition deficits in mice after phencyclidine administration. Clin. Psychopharmacol. Neurosci. 10, 94-98. doi: 10.9758/cpn.2012.10.2.94

Shirai, Y., Fujita, Y., Hashimoto, R., Ohi, K., Yamamori, H., Yasuda, Y., et al. (2015). Dietary intake of sulforaphane-rich broccoli sprout extracts during juvenile and adolescence can prevent phencyclidine-induced cognitive deficits at adulthood. PLoS One 10:e0127244. doi: 10.1371/journal. pone. 0127244

Steinert, C., Hofmann, M., Kruse, J., and Leichsenring, F. (2014). Relapse rates after psychotherapy for depression-Stable long-term effects? A meta-analysis. J. Affect. Disord. 168, 107-118. doi: 10.1016/j.jad.2014.06.043

Steullet, P., Cabungcal, J. H., Monin, A., Dwir, D., O’Donnell, P., Cuenod, M., et al. (2016). Redox dysregulation, neuroinflammation, and NMDA receptor hypofunction: a "central hub" in schizophrenia pathophysiology? Schizophr. Res. 176, 41-51. doi: 10.1016/j.schres.2014.06.021

Strawbridge, R., Arnone, D., Danese, A., Papadopoulos, A., Herane Vives, A., and Cleare, A. J. (2015). Inflammation and clinical response to treatment in depression: a meta-analysis. Eur. Neuropsychopharmacol. 25, 1532-1534. doi: 10.1016/j.euroneuro.2015.06.007

Swardfager, W., Hennebelle, M., Yu, D., Hammock, B. D., Levitt, A. J., Hashimoto, K., et al. (2018). Tetabbolic/inflammatory/vascular comorbidity in psychiatric disorders; soluble epoxide hydrolase $(\mathrm{sEH})$ as a possible new target. Neurosci. Biobehav. Rev. 87, 56-66. doi: 10.1016/j.neubiorev.2018.01.010

Upthegrove, R., Manzanares-Teson, N., and Barnes, N. M. (2014). Cytokine function in medication-naïve first episode psychosis: a systematic review and meta-analysis. Schizophr. Res. 155, 101-108. doi: 10.1016/j. schres.2014.03.005

van Kesteren, C. F., Gremmels, H., de Witte, L. D., Hol, E. M., Van Gool, A. R., Falkai, P. G., et al. (2017). Immune involvement in the pathogenesis of schizophrenia: a meta-analysis on postmortem brain studies. Transl. Psychiatry 7:e1075. doi: 10.1038/tp.2017.4

Wagner, K. M., McReynolds, C. B., Schmidt, W. K., and Hammock, B. D. (2017). Soluble epoxide hydrolase as a therapeutic target for pain, inflammatory and neurodegenerative diseases. Pharmacol. Ther. 180, 62-76. doi: 10.1016/j. pharmthera.2017.06.006

Wagner, K., Vito, S., Inceoglu, B., and Hammock, B. D. (2014). The role of long chain fatty acids and their epoxide metabolites in nociceptive signaling. Prostaglandins Other Lipid Mediat. 113-115, 2-12. doi: 10.1016/j. prostaglandins.2014.09.001

Wang, D. F., Sun, X. Y., Yan, J. J., Ren, B., Cao, B., Lu, Q. B., et al. (2018). Alterations of eicosanoids and related mediators in patients with schizophrenia. J. Psychiatr. Res. 102, 168-178. doi: 10.1016/j.jpsychires.2018.04.002

World Health Organization. (2018a). Depression. 22 March 2018. Available at: http://www.who.int/news-room/fact-sheets/detail/depression (Accessed November 3, 2018).

World Health Organization. (2018b). Schizophrenia. 9 April 2018. Available at: http://www.who.int/news-room/fact-sheets/detail/schizophrenia (Accessed November 3, 2018).

Wu, Q., Cai, H., Song, J., and Chang, Q. (2017). The effects of sEH inhibitor on depression-like behavior and neurogenesis in male mice. J. Neurosci. Res. 95, 2483-2492. doi: 10.1002/jnr.24080

Wu, Q., Song, J., Meng, D., and Chang, Q. (2019). TPPU, a sEH inhibitor, attenuates corticosterone-induced PC12 cell injury by modulation of BDNF-TrkB pathway. J. Mol. Neurosci. 67:364. doi: 10.1007/s12031-018-1230-z

Yao, J. K., and Keshavan, M. S. (2011). Antioxidants, redox signaling, and pathophysiology in schizophrenia: an integrative view. Antioxid. Redox Signal. 15, 2011-2035. doi: 10.1089/ars.2010.3603

Young, J. J., Bruno, D., and Pomara, N. (2014). A review of the relationship between proinflammatory cytokines and major depressive disorder. J. Affect. Disord. 169, 15-20. doi: 10.1016/j.jad.2014.07.032

Zhang, G., Kodani, S., and Hammock, B. D. (2014). Stabilized epoxygenated fatty acids regulate inflammation, pain, angiogenesis and cancer. Prog. Lipid Res. 53, 108-123. doi: 10.1016/j.plipres.2013.11.003

Zhang, J. C., Wu, J., Fujita, Y., Yao, W., Ren, Q., Yang, C., et al. (2015). Antidepressant effects of TrkB ligands on depression-like behavior and dendritic changes in mice after inflammation. Int. J. Neuropsychopharmacol. 18:pyu077. doi: 10.1093/ijnp/pyu077

Zhang, J. C., Yao, W., and Hashimoto, K. (2016). Brain-derived neurotrophic factor (BDNF)-TrkB signaling in inflammation-related depression and potential therapeutic targets. Curr. Neuropharmacol. 14, 721-731. doi: 10.2174/157015 9X14666160119094646

Conflict of Interest Statement: The author declares that the research was conducted in the absence of any commercial or financial relationships that could be construed as a potential conflict of interest.

Copyright (c) 2019 Ren. This is an open-access article distributed under the terms of the Creative Commons Attribution License (CC BY). The use, distribution or reproduction in other forums is permitted, provided the original author(s) and the copyright owner(s) are credited and that the original publication in this journal is cited, in accordance with accepted academic practice. No use, distribution or reproduction is permitted which does not comply with these terms. 